TRANSACTIONS OF THE

AMERICAN MATHEMATICAL SOCIETY

Volume 331, Number 1, May 1992

\title{
GAUGE INVARIANT QUANTIZATION ON RIEMANNIAN MANIFOLDS
}

\author{
LIU ZHANG-JU AND QIAN MIN
}

\begin{abstract}
For every pointwise polynomial function on each fiber of the cotangent bundle of a Riemannian manifold $M$, a family of differential operators is given, which acts on the space of smooth sections of a vector bundle on $M$. Such a correspondence may be considered as a rule to quantize classical systems moving in a Riemannian manifold or in a gauge field. Some applications of our construction are also given in this paper.
\end{abstract}

\section{INTRODUCTION}

How to quantize classical Hamiltonian systems with symplectic manifolds as the phase space is a very important problem in which mathematicians as well as physicists are interested. Several mathematical methods have been developed to solve this problem, e.g., geometric quantization, deformation theory, functional integration and Maslov's theory (see [2, 3, 4, 6, 10, 11, 14]). In cases when the phase space is flat, the quantization rules are more or less established. But when the phase space is $T^{*} M$, the cotangent bundle of a Riemannian manifold $M$, different quantization approaches lead to different results even for the simplest case of geodesic flow in $M$ with the Hamiltonian

$$
H(x, y)=g_{x}(y, y), \quad x \in M, y \in T_{x}^{*} M,
$$

where $g$ denotes the Riemannian metric of $M$. But the quantized Hamiltonians always take the following form

$$
\widehat{H}=-\hbar^{2}(\Delta+\alpha R),
$$

where $\hbar>0$ is the Planck constant, $\Delta$ and $R$ denote the Laplace-Beltrami operator and the scalar curvature respectively with respect to the Riemannian connection of $(M, g)$, and the constant $\alpha$ depends on the way of quantization.

In this paper we shall develop a method of quantization on Riemannian manifolds, by means of the Weyl transformation together with some locally canonical transformation, which means symplectic in the classical case and unitary in the quantum case. In $\S 3$, we extend our quantization procedure to a principal bundle, so it can be applied to quantize classical particle systems moving in Yang-Mills fields [5], with gauge invariance also considered. In the last section, we briefly mention two applications of the quantization rule given by us. One is about the semiclassical approximation for a quantized system,

Received by the editors May 18, 1989 and, in revised form, February 22, 1990.

1980 Mathematics Subject Classification (1985 Revision). Primary 81D07. 
and the other is concerned with quantum integrable systems constrained on the sphere; the details may be found in [7 and 8]. Only pointwise polynomial functions are quantized here in order to get differential operators. We know other mathematicians have already set up the symbol calculus for pseudo-differential operators on Riemannian manifolds, but pay no attention to the physical aspect (e.g., see [12]). The differences-both in the results obtained and in the point of view-are also pointed out in the last section.

We end this introduction with a sketch of the quantization procedure on flat spaces. Let $\left(x^{1}, \ldots, x^{n}, p_{1}, \ldots, p_{n}\right)$ be the canonical coordinates of $\mathbb{R}^{2 n}$ and $L^{2}\left(\mathbb{R}_{x}^{n}, d x\right)$ the space of quantum wave functions. The canonical quantization of basic observables is that

$$
x^{j} \rightarrow \hat{x}^{j} ; \quad p_{j} \rightarrow \hat{p}_{j}=-\hbar i \frac{\partial}{\partial x^{j}},
$$

where $\hat{x}^{j}$ is the multiplication operator. Most generally, observables could be quantized in different orders [13]:

$$
\begin{array}{lrl}
\hat{f}_{N}=\int \tilde{f}(\alpha, \beta) e^{i\langle\alpha, \hat{x}\rangle} e^{i\langle\beta, \hat{p}\rangle} d \alpha d \beta & \text { (normal), } \\
\hat{f}_{W}=\int \tilde{f}(\alpha, \beta) e^{i(\langle\alpha, \hat{x}\rangle+\langle\beta, \hat{p}\rangle)} d \alpha d \beta & \text { (Weyl), } \\
\hat{f}_{A}=\int \tilde{f}(\alpha, \beta) e^{i\langle\beta, \hat{p}\rangle} e^{i\langle\alpha, \hat{x}\rangle} d \alpha d \beta & \text { (antinormal), }
\end{array}
$$

where $\tilde{f}$ is the Fourier transformation of $f$. It is well known that

$f \in L^{2}\left(\mathbb{R}^{2 n}, d x d p\right) \Leftrightarrow \hat{f}_{W}$ is a Hilbert-Schimit operator.

$f$ is a polynomial of $p \Leftrightarrow \hat{f}$ is a differential operator,

The above-mentioned quantization rule can be expressed in a unified form by introducing an order parameter $\lambda$.

Definition. For any $\lambda \in[0,1]$, the $\lambda$-Weyl transformation is defined as

$$
\hat{f}_{\lambda}=\int \tilde{f}(\alpha, \beta) e^{i \hbar(\lambda-1 / 2)\langle\alpha, \beta\rangle} e^{i(\langle\alpha, \hat{x}\rangle+\langle\beta, \hat{p}\rangle)} d \alpha d \beta
$$

in which $\hat{f}_{0}=\hat{f}_{N}, \hat{f}_{1 / 2}=\hat{f}_{W}$, and $\hat{f}_{1}=\hat{f}_{A}$. The operator defined above acts on $\phi \in C_{0}^{\infty}\left(\mathbb{R}_{x}^{n}\right)$ as

$$
\left(\hat{f}_{\lambda} \phi\right)(x)=\hbar^{-n} \int f(x+\lambda \beta, p) e^{-i / \hbar\langle\beta, p\rangle} \phi(x+\beta) d \beta d p .
$$

The expression $(*)$ will be used in the next section, and from $(*)$, it is obvious that

$$
\widetilde{\left(x^{j} p_{j}\right)_{\lambda}}=\lambda \hat{p}_{j} \hat{x}^{j}+(1-\lambda) \hat{x}^{j} \hat{p}_{j}
$$

\section{Definition of $\lambda$-WeYl tRANSFORMATION ON RIEMANNIAN MANIFOLdS}

Assume $(M, g)$ to be a $n$-dimensional oriented Riemannian manifold. In this section, we do not consider the gauge invariance. Thus we take $L^{2}(M, d \mu)$ (complex-valued) as the space of quantum wave functions, where $d \mu$ is the positive Riemannian measure induced by $g$. The construction of $\lambda$-Weyl transformation may be split into four steps: 
Step 1 (pulling classical systems to flat space by a local symplectic transformation in a neighborhood of a point). For a fixed point $x \in M$, let $B_{2 \varepsilon}$ be the $2 \varepsilon$-ball in $T_{x} M$ with zero as center, where $\varepsilon$ is taken so small that the exponential map $\exp _{x}: B_{2 \varepsilon} \rightarrow U_{2 \varepsilon} \subset M$ is a diffeomorphism, where $U_{2 \varepsilon}$ is the $2 \varepsilon$-geodesic ball neighborhood of $x$. It is known that the map $\exp _{x}$ induces a symplectic diffeomorphism $\exp _{x}^{*}$ from $T^{*} B_{2 \varepsilon}=B_{2 \varepsilon} \times T_{x}^{*} M$ onto $T^{*} U_{2 \varepsilon}$, where both symplectic structures are induced by the natural symplectic structure $\omega$ of $T^{*} M$. By use of this canonical transformation any classical observable in $T^{*} M$ can be locally considered as in $B_{2 \varepsilon} \times T_{x}^{*} M$ and can be extended to $T_{x} M \times T_{x}^{*} M$ by multiplying a cut-off function $X_{\varepsilon}\left(X_{\varepsilon} \equiv 1\right.$ inside $B_{\varepsilon}, X_{\varepsilon}=0$ outside $\left.B_{2 \varepsilon}\right)$. That is, for any $f \in C^{\infty}\left(T^{*} M\right)$, we can get a function $\tilde{f}_{x} \in C^{\infty}\left(T_{x} M \times T_{x}^{*} M\right)$ defined by

$$
\tilde{f}_{x}(\beta, p)=X_{\varepsilon}(\beta) f \circ \exp _{x}^{*}(\beta, p) .
$$

Step 2 (quantization of $T_{x} M \times T_{x}^{*} M$ ). We can get a operator $\hat{\tilde{f}}_{x, \lambda}$ by quantizing $\tilde{f}_{x}$ with order $\lambda$ defined in $\S 0$. In general, $\hat{\tilde{f}}_{x, \lambda}$ depends on the choice of $X_{\varepsilon}$. But we have

Lemma 1.1. If $f$ is a pointwise polynomial on each fiber of $T^{*} M$, then, for any $\phi \in C^{\infty}\left(T_{x} M\right),\left.\left(\hat{\tilde{f}}_{x, \lambda} \varphi\right)\right|_{B_{\varepsilon}}$ is independent of the choice of $X_{\varepsilon}$.

Proof. Since the transformation $\exp _{x}^{*}$ is linear on each fiber, $\tilde{f}_{x}$ is also a polynomial of $p$, then $\hat{\tilde{f}}_{x, \lambda}$ is a differential operator, i.e., a local operator. The lemma is true because $\left.X_{\varepsilon}\right|_{B_{\varepsilon}}=1$.

In this case, $\hat{\tilde{f}}_{x, \lambda}$ is a well-defined differential operator on $L^{2}\left(B_{\varepsilon}, d \mu_{x}\right)$ where $d \mu_{x}$ is the Lebesgue measure on $T_{x} M$ induced by the Riemannian metric $g_{x}$.

Step 3 (pulling $\hat{\tilde{f}}_{x, \lambda}$ back to $L^{2}\left(U_{\varepsilon},\left.d \mu\right|_{U_{\varepsilon}}\right)$ by a unitary transformation). Since both $\left.d \mu\right|_{U_{\varepsilon}}$ and $D^{*} \exp _{x}^{-1}\left(d \mu_{x}\right)$ are positive $n$-forms on $U_{\varepsilon}$, there exists a unique positive function $\rho_{x}$ defined on $U_{\varepsilon}$ such that $\rho_{x} D^{*} \exp _{x}^{-1}\left(d \mu_{x}\right)=$ $\left.d \mu\right|_{U_{\varepsilon}}$. We have

Lemma 1.2. The transformation $K_{x}$ defined by

$$
\left(K_{x} \phi\right)(\beta)=\rho_{x}^{1 / 2}\left(\exp _{x} \beta\right) \phi\left(\exp _{x} \beta\right)
$$

is unitary from $L^{2}\left(U_{\varepsilon},\left.d \mu\right|_{U_{\varepsilon}}\right)$ to $L^{2}\left(B_{\varepsilon}, d \mu_{x}\right)$.

The proof of Lemma 1.2 is trivial. Consequently, we get a differential operator on $L^{2}\left(U_{\varepsilon},\left.d \mu\right|_{U_{\varepsilon}}\right)$ defined by

$$
\hat{f}_{x, \lambda}=K_{x}^{-1} \circ \hat{\tilde{f}}_{x, \lambda} \circ K_{x}
$$

which is independent of choice of $X_{\varepsilon}$.

Step 4 (from local to global). $f$ and $\hat{f}_{x, \lambda}$ locally give the correspondence between classical and quantum observables since every step in the construction is canonical. But, in general, $\hat{f}_{x, \lambda}$ is dependent on the choice of $x \in U_{\varepsilon}$, unless $M$ has some symmetric properties (e.g., $M=S^{n}$ ). This is not unreasonable 
because different local trivializations just mean different gauges for the local representation of observables. The only way to get a global invariant operator is to define it point by point on $M$. It is known that the above process is reasonable only for local operators. In our case, $\hat{f}_{x, \lambda}$ is a differential operator which is certainly local, therefore, we have

Definition 1.1. Let $f \in C^{\infty}\left(T^{*} M\right)$ be a pointwise polynomial on each fiber. The $\lambda$-Weyl transformation of $f$ is defined as a differential operator $\hat{f}_{\lambda}$ on $L^{2}(M, d \mu)$ as follows:

$$
\left(\hat{f}_{\lambda} \phi\right)(x)=\left(\hat{f}_{x, \lambda} \phi\right)(x)=\left(K_{x}^{-1} \circ \hat{\tilde{f}}_{x, \lambda} \circ K_{x} \phi\right)(x)
$$

where $\phi \in C^{\infty}(M)$.

$$
\begin{array}{r}
\text { Writing } A(\beta)=D^{*} \exp _{x}^{-1}(\beta): T_{x}^{*} M \rightarrow T_{\exp _{x} \beta}^{*} M \text {, i.e., } \\
\qquad \exp _{x}^{*}(\beta, p)=\left(\exp _{x} \beta, A(\beta) p\right)
\end{array}
$$

and using the expression $(*)$ in $\S 0$, the above definition can be made more explicit as

$$
\begin{array}{rl}
\left(\hat{f}_{\lambda} \phi\right)(x)=h^{-n} \int_{T_{x} M \times T_{x}^{*} M} & f\left(\exp _{x}(\lambda \beta), A(\lambda \beta) p\right) \\
& \times e^{-i / h\langle\beta, p\rangle} \cdot \phi_{\varepsilon}\left(\exp _{x} \beta\right) \cdot \rho_{x}^{1 / 2}\left(\exp _{x} \beta\right) d \beta d p .
\end{array}
$$

Remarks. (1) The fact $\rho_{x}(x)=1$ has been used in (1.1).

(2) $\phi_{\varepsilon}=x_{\varepsilon} \phi$, but actually (1.1) is independent of $X_{\varepsilon}$.

(3) When $M=\mathbb{R}^{n},(1.1)=(*)$ because $A=\mathrm{id}, \rho_{x} \equiv 1$ and $\exp _{x} \lambda \beta=$ $x+\lambda \beta$ in this case.

Since the most interesting case is that degree $f=2$, we give a result as follows

Theorem 1.1. If $f=\langle X, p \otimes p\rangle=X^{i j}(x) p_{i} p_{j}$ where $X$ is a smooth 2-symmetric contravariant tensor field on $M$, then

$$
\hat{f}_{\lambda}=-h^{2}\left(X^{i j} \nabla_{i} \nabla_{j}+2 \lambda \nabla_{i} X^{i j} \nabla_{j}+\lambda^{2} \nabla_{i} \nabla_{j} X^{i j}-\frac{2 \lambda^{2}+1}{6} X^{i j} R_{i j}\right)
$$

where $\nabla_{i}$ is the covariant derivative with respect to the Levi-Civita connection and $\left\{R_{i j}\right\}$ is the Ricci curvature tensor. In particular, if $f=\frac{1}{2}\|p\|^{2}=\frac{1}{2} g^{i j} p_{i} p_{j}$,

$$
\begin{array}{lr}
\hat{f}_{0}=-\frac{h^{2}}{2} \Delta+\frac{h^{2}}{12} R & (\text { normal }), \\
\hat{f}_{1 / 2}=-\frac{h^{2}}{2} \Delta+\frac{h^{2}}{8} R & (\text { Weyl }), \\
\hat{f}_{1}=-\frac{h^{2}}{2} \Delta+\frac{h^{2}}{4} R & (\text { antinormal }),
\end{array}
$$

where $\Delta$ is the Laplace-Beltrami operator on $M$ and $R$ is the scalar curvature.

Remark. It is well known that the scalar curvature $R$ should appear by quantizing $\frac{1}{2}\|p\|^{2}$ on a Riemannian manifold. But the coefficient factor is different according to different quantization approaches, e.g., $\frac{R}{6}, \frac{R}{12}$ or zero by pathintegrations, $\frac{R}{12}$ by geometric quantization [14] and $\frac{R}{4}$ by Maslov's theory [11].

We fix the notation as follows: For a fixed $x \in M,\left(\beta^{1}, \ldots, \beta^{n}\right)$ is the normal coordinate at $x$ with respect to which $\left\{\Gamma_{j k}^{i}\right\}$ are the coefficients of connection. To prove Theorem 1.1, we first prove three lemmas. 
Lemma 1.3. For $f=X^{i_{1} \cdots i_{k}} p_{i_{1}} \cdots p_{i_{k}}$, the following formula holds:

$$
\begin{aligned}
\left(\hat{f}_{\lambda} \phi\right)(x)=\left(\frac{h}{i}\right)^{k} \frac{\partial^{k}}{\partial \beta^{i_{1}} \cdots \partial \beta^{i_{k}}}\left(X^{j_{1} \cdots j_{k}}(\lambda \beta) A_{j_{1}}^{i_{1}}(\lambda \beta)\right. & \\
\left.\cdots A_{j_{k}}^{i_{k}}(\lambda \beta) \phi(\beta) \rho_{x}^{1 / 2}(\beta)\right)\left.\right|_{\beta=0} & .
\end{aligned}
$$

Proof. From (1.1), one has

$$
\begin{aligned}
\left(\hat{f}_{\lambda} \phi\right)(x)= & h^{k} \int X^{j_{1} \cdots j_{k}}(\lambda \beta) A_{j_{1}}^{i_{1}}(\lambda \beta) \cdots A_{j_{k}}^{i_{k}}(\lambda \beta) p_{i_{1}} \\
& \cdots p_{i_{k}} e^{-i \beta^{j} p_{j}} \phi_{\varepsilon}(\beta) \rho_{x}^{1 / 2}(\beta) d \beta d p . \\
= & \frac{h^{k}}{(-i)^{k}} \int X^{j_{1} \cdots j_{k}} A_{j_{1}}^{i_{1}} \cdots A_{j_{k}}^{i_{k}} \phi_{\varepsilon} \rho_{x}^{1 / 2} \frac{\partial^{k}}{\partial \beta^{i_{1}} \cdots \partial \beta^{i_{k}}} e^{-i \beta^{j} p_{j}} d \beta d p \\
= & \left(\frac{h}{i}\right)^{k} \int e^{i \beta^{j} p_{j}} \frac{\partial^{k}}{\partial \beta^{i_{1}} \cdots \partial \beta^{i_{k}}}\left(X^{j_{1} \cdots j_{k}} A_{j_{1}}^{i_{1}} \cdots A_{j_{k}}^{i_{k}} \phi_{\varepsilon} \rho_{x}^{1 / 2}\right) d \beta d p .
\end{aligned}
$$

Thus, formula (1.2) is obtained by using Fourier transform to (1.3).

Lemma 1.4. With the notation above, one has the formula

$$
\frac{\partial^{2} \operatorname{tr} A(0)}{\partial \beta^{i} \partial \beta^{j}}=\frac{\partial^{2} \operatorname{det} A(0)}{\partial \beta^{i} \partial \beta^{j}}=\left.\frac{1}{3}\left(\frac{\partial \Gamma_{i j}^{k}}{\partial \beta^{k}}+\frac{\partial \Gamma_{j k}^{k}}{\partial \beta^{j}}+\frac{\partial \Gamma_{j k}^{k}}{\partial \beta^{i}}\right)\right|_{\beta=0} .
$$

Proof. Writing $D(\beta)=D_{*} \exp _{x} \beta$, then $A(\beta)=D^{-1}(\beta)$. Obviously, $D(0)=$ id and $\partial D_{j}^{i}(0) / \partial \beta^{k}=0$ for $k=1, \ldots, n$. Consequently, for $\beta$ small enough, we have

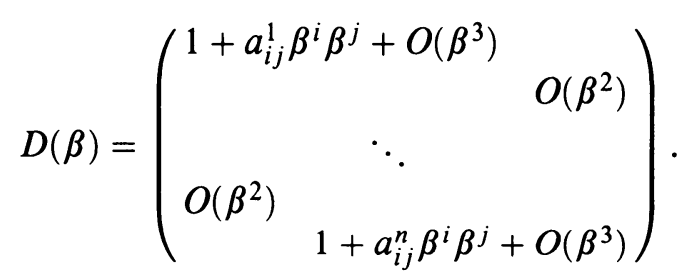

From (1.4), it is obvious that

$$
\frac{\partial^{2} \operatorname{det} D(0)}{\partial \beta^{i} \partial \beta^{j}}=\frac{\partial^{2} \operatorname{tr} D(0)}{\partial \beta^{i} \partial \beta^{j}}=\sum_{k=1}^{n} a_{i j}^{k}
$$

where $a_{i j}^{k}$ are constants determined by the curvature tensor at $x .(1.5)$ is also true for $A$ because $A=D^{-1}$.

Setting $y=\exp _{x} s \beta_{0}$ for a fixed $s \in R$, notice that $x(t)=\exp _{x}(t+s) \beta_{0}$ is a geodesic starting at $y$, therefore, it satisfies the geodesic equation. In particular at $y$, we have

$$
\left.\left(\frac{d^{2} x^{i}}{d t^{2}}+\Gamma_{j_{1} j_{2}}^{i}\left(s \beta_{0}\right) \frac{d x^{j_{1}}}{d t} \frac{d x^{j_{2}}}{d t}\right)\right|_{t=0}=0
$$

i.e.,

$$
\left.\left(\frac{\partial x^{i}}{\partial \beta^{l_{1}} \partial \beta^{l_{2}}} \beta_{0}^{l_{1}} \beta_{0}^{l_{2}}+\Gamma_{j_{1} j_{2}}^{i}\left(s \beta_{0}\right) \frac{\partial x^{j_{1}}}{\partial \beta^{l_{1}}} \frac{\partial x^{j_{2}}}{\partial \beta^{l_{2}}}\right)\right|_{s \beta_{0}}=0
$$


Differentiating (1.6) at $s=0$ and using $D_{j}^{i}(0)=\partial x^{i}(0) / \partial \beta^{j}=\delta_{j}^{i}$, one has

$$
\frac{\partial^{3} x^{i}(0)}{\partial \beta^{l_{1}} \partial \beta^{l_{2}} \partial \beta^{l_{3}}} \beta_{0}^{l_{1}} \beta_{0}^{l_{2}} \beta_{0}^{l_{3}}+\frac{\partial \Gamma_{j_{1} j_{2}}^{i}(0)}{\partial \beta^{j_{3}}} \beta_{0}^{j_{1}} \beta_{0}^{j_{2}} \beta_{0}^{j_{3}}=0 .
$$

Because the choice of $\beta_{0}$ is arbitrary, the final result is

$$
\frac{\partial^{2} \operatorname{tr} D(0)}{\partial \beta^{i} \partial \beta^{j}}=\frac{\partial^{3} x^{k}(0)}{\partial \beta^{i} \partial \beta^{j} \partial \beta^{k}}=-\left.\frac{1}{3}\left(\frac{\partial \Gamma_{j k}^{k}}{\partial \beta^{j}}+\frac{\partial \Gamma_{j k}^{k}}{\partial \beta^{i}}+\frac{\partial \Gamma_{i j}^{k}}{\partial \beta^{k}}\right)\right|_{\beta=0} \text {. }
$$

From (1.5), (1.7) and the fact that $\operatorname{det} A=\operatorname{det}^{-1} D$, i.e.,

$$
\frac{\partial^{2} \operatorname{det} A(0)}{\partial \beta^{i} \partial \beta^{j}}=\frac{-\partial^{2} \operatorname{det} D(0)}{\partial \beta^{i} \partial \beta^{j}}
$$

them lemma is obviously true.

Lemma 1.5. The following formulas hold:

(a) $\rho_{x}(x)=1$,

(b) $\partial \rho_{x}(0) / \partial \beta^{i}=0$,

(c) $\partial^{2} \rho_{x}(0) / \partial \beta^{i} \partial \beta^{j}=R_{i j}(x)$.

Proof. (a), (b) are obvious. To prove (c) note that, in normal coordinates, one has

$$
\rho_{x}(y)=g^{1 / 2}(y) \operatorname{det} D(\beta)
$$

where $y=\exp _{x} \beta, g=\operatorname{det}\left(g_{i j}\right)$, and

$$
\frac{\partial^{2} g^{1 / 2}(0)}{\partial \beta^{i} \partial \beta^{j}}=\frac{\partial \Gamma_{j k}^{k}}{\partial \beta^{i}}, \quad R_{i j}(x)=\frac{\partial \Gamma_{i j}^{k}}{\partial \beta^{k}}-\frac{\partial \Gamma_{i k}^{k}}{\partial \beta^{j}} .
$$

From these facts and Lemma 1.4, it is not difficult to get Lemma 1.5.

The proof of Theorem 1.1 follows from Lemmas 1.3, 1.4, 1.5 and the facts that, in normal coordinates,

$$
\nabla_{i} \nabla_{j} X^{i j}(x)=\frac{\partial X^{i j}(0)}{\partial \beta^{i} \partial \beta^{j}}+X^{i j}\left(\frac{\partial \Gamma_{i k}^{k}}{\partial \beta^{j}}+\frac{\partial \Gamma_{i j}^{k}}{\partial \beta^{k}}\right)
$$

and

$$
\frac{\partial^{2} A_{j}^{i}(0)}{\partial \beta^{k} \partial \beta^{j}}=\frac{\partial^{2} A_{j}^{j}(0)}{\partial \beta^{k} \partial \beta^{i}}=\frac{\partial^{2} \operatorname{tr} A(0)}{\partial \beta^{k} \partial \beta^{i}}
$$

\section{SOME PROPERTIES OF $\lambda$-WEYL TRANSFORMATIONS}

In this section, we are going to show some properties of $\lambda$-Weyl transformations.

Theorem 2.1 (Wigner's formula). With the same notation as in $\S 1$ and $(\cdot, \cdot)$ expressing the inner-product in the Hilbert space $L^{2}(M, d \mu)$, then, for any $\phi$, $\psi \in C_{0}^{\infty}(M)$ we have

$$
\left(\hat{f}_{\lambda} \phi, \psi\right)=\int_{T^{*} M} f(x, p) \Phi_{\lambda}(x, p) d w
$$


where $d w=d x d p$ is the symplectic volume element of $T^{*} M$ and

$$
\begin{aligned}
\Phi_{\lambda}(x, p)=h^{-n} \int_{T_{x} M} \phi_{\varepsilon}\left(\exp _{x}(1-\lambda) \beta\right) \bar{\psi}_{\varepsilon}\left(\exp _{x}-\lambda \beta\right) \\
\cdot e^{-i / h\langle\beta, p\rangle} \bar{\rho}_{\lambda}(x, \beta) d \beta
\end{aligned}
$$

where

$$
\bar{\rho}_{\lambda}(x, \beta)=\rho_{\exp _{x}-\lambda \beta}^{1 / 2}(\exp (1-\lambda) \beta) \rho_{x}\left(\exp _{x}-\lambda \beta\right) .
$$

Remarks. (1) Formula (2.1) is well defined because $\Phi_{\lambda} \in S$ along each fiber where $S$ is the Schwartz space.

(2) When $M=\mathbb{R}^{n}, \Phi_{1 / 2}$ is the usual Wigner function [13].

(3) (2.1) can be taken as the weak definition of $\lambda$-Weyl transformation on Riemannian manifolds.

Proof. Take $\varepsilon \ll 1$ such that, for any $x \in \operatorname{Supp} \psi, \exp _{x}: B_{\varepsilon}(x) \rightarrow U_{\varepsilon}(x)$ is one-to-one. Notice that

(a) Since $\exp _{x}^{*}: B_{\varepsilon}(x) \times T_{x}^{*} M \rightarrow T^{*} U_{\varepsilon}(x)$ is symplectic we have $d \beta d p=$ $D^{*} \exp _{x}^{*}\left(d w \mid T^{*} U_{\varepsilon}\right)$.

(b) By the expression $\exp _{x}^{*}(\beta, p)=\left(\exp _{x} \beta, A(\beta) p\right)$, we can write $y=$ $\exp _{x} \lambda \beta, \beta^{\prime}=D_{*} \exp _{x}(\lambda \beta)$ and $p^{\prime}=A(\lambda \beta) p$, i.e., $\left(\beta^{\prime}, p^{\prime}\right) \in T_{y} M \times T_{y}^{*} M$. By Gauss' lemma, $\beta^{\prime}=\tau(x, y) \beta$, where $\tau(x, y)$ is the parallel displacement from $x$ to $y$ along the geodesic $Y(t)=\exp _{x} t \lambda \beta$. Consequently, we have $\left\langle\beta^{\prime}, p^{\prime}\right\rangle=\langle\beta, p\rangle, \exp _{x} \beta=\exp _{y}(1-\lambda) \beta^{\prime}$, and $x=\exp _{y}-\lambda \beta^{\prime}$.

(c) From (b) and the definition of the function $\rho_{y}$, we have $d \mu(x)=$ $\rho_{y}\left(\exp _{y}-\lambda \beta^{\prime}\right) d \beta^{\prime}$, where $d \mu(x)$ is the Riemannian measure at $x$ and $d \beta^{\prime}$ is the Lebesgue measure on $T_{y} M$ induced by Riemannian metric $g_{y}$.

Now, by the definition of $\hat{f}_{\lambda}$, one has

$$
\left(\hat{f}_{\lambda} \phi, \psi\right)=\int_{M} \int_{T_{x} M \times T_{x}^{*} M}(\cdot) d \beta d p d \mu(x)
$$

where $(\cdot)$ is an abbreviation for the integrand. Writing $d w=d y d p^{\prime}$, we have

$$
\begin{aligned}
&\left(\hat{f}_{\lambda} \phi, \psi\right) \stackrel{\text { (by (a)) }}{=} \int_{M} \int_{T^{*} U_{\varepsilon}(x)}(\cdot) d y d p^{\prime} d \mu(x) \\
&=\int_{T^{*} M} \int_{U_{\varepsilon}(y)}(\cdot) d \mu(x) d y d p^{\prime} \\
& \stackrel{(\text { by (c)) })}{=} \int_{T^{*} M}\left[\int_{T_{y} M}(\cdot) \rho_{y}\left(\exp _{y}-\lambda \beta^{\prime}\right) d \beta^{\prime}\right] d w \\
&=\int_{T^{*} M} f\left(y, p^{\prime}\right) \Phi_{\lambda}\left(y, p^{\prime}\right) d w .
\end{aligned}
$$

The last equation holds by (b) and the expression of $(\cdot)$, where $\Phi_{\lambda}$ is actually the Wigner function defined in (2.2).

Notice that, when $M=R^{n}$, the Weyl's ordered quantization $\hat{f}_{1 / 2}$ is always a symmetric operator, which can be easily verified by the symmetric property of $\Phi_{1 / 2}$, i.e., $\Phi_{1 / 2}(\phi, \psi)=\Phi_{1 / 2}(\psi, \phi)$ (in this case, $\bar{\rho}_{1 / 2}=1$ ). In general, $\Phi_{1 / 2}$ defined in (2.2) has no such symmetrical property because there appears a nonsymmetric factor $\bar{\rho}_{1 / 2}(x, \beta)$ unless $M$ has some symmetrical properties such that $\bar{\rho}_{1 / 2}(x, \beta)=\bar{\rho}_{1 / 2}(x,-\beta)$. But we can get 
Theorem 2.2. (1) $\hat{f}_{1 / 2}$ is symmetric if $\operatorname{deg} f \leq 2$.

(2) When $M$ is a constant curvature space, $\hat{f}_{1 / 2}$ is symmetric for all polynomials $f$.

Remark. In case $M$ is compact, a symmetric operator is actually selfadjoint (a quantum observable).

Proof. From Lemma 1.3, we can see that, if degree $f=k$, only derivatives (for $\beta$ ) of degree $\leq k$ affect $\hat{f}_{\lambda}$. Consequently, if we expand $\bar{\rho}_{1 / 2}(x, \beta)=$ $\bar{\rho}_{1 / 2}^{k}(x, \beta)+O\left(\beta^{k+1}\right)$, we can show that $\hat{f}_{1 / 2}$ is symmetric if $\bar{\rho}_{1 / 2}^{k}$ is symmetric, i.e., if $\bar{\rho}^{k}(x, \beta)=\bar{\rho}^{k}(x,-\beta)$ in $B_{\varepsilon}$. From the analysis in Lemma 1.5 , we see that $\bar{\rho}_{1 / 2}(\beta)=1+b_{i j} \beta^{i} \beta^{j}+O\left(\beta^{3}\right)$, where the constants $b_{i j}$ are determined by the Ricci curvature tensor at $x$, so the lemma is true in case (1). When $M$ is a constant curvature space, the coefficients of $\beta^{k}, k \geq 3$, are always zero because they are determined by the covariant derivatives of the curvature tensor which are zero in this case, so (2) is true.

Let $P^{1}=$ polynomials of degree $\left.\leq 1\right\} .\left(P^{1},\{\cdot, \cdot\}\right)$ forms a Lie algebra which is a generalization of Heisenberg Lie algebra, where $\{\cdot, \cdot\}$ is Poisson bracket. From Lemma 1.3, we see that, if $\operatorname{deg} f=0, \hat{f}_{\lambda}=f$ is a multiplication operator. If $\operatorname{deg} f=1$, i.e., $f=\langle X, p\rangle=X^{i} p_{i}$, then

$$
\hat{f}_{\lambda}=\frac{h}{i}\left(X^{i} \nabla_{i}+\lambda \nabla_{i} X^{i}\right)=\frac{h}{i}\left(\nabla_{X}+\lambda \operatorname{div} X\right) .
$$

Theorem 2.3. The $\lambda$-Weyl transformation is a representation of the Lie algebra $\left(P^{1},\{\cdot, \cdot\}\right)$, i.e., for $f, g \in P^{1}$, we have

$$
i h\{\widehat{f, g}\}_{\lambda}=\hat{f}_{\lambda} \hat{g}_{\lambda}-\hat{g}_{\lambda} \hat{f}_{\lambda}=\left[\hat{f}_{\lambda}, \hat{g}_{\lambda}\right] \text {. }
$$

Theorem 2.3. The $\lambda$-Weyl transformation is a representation of the Lie algebra $\left(P^{1},\{\cdot, \cdot\}\right)$, i.e., for $f ; g \in P^{1}$, we have

$$
\operatorname{ih}\{\widehat{f, g}\}_{\lambda}=\hat{f}_{\lambda} \hat{g}_{\lambda}-\hat{g}_{\lambda} \hat{f}_{\lambda}=\left[\left\langle\hat{f}_{\lambda}, \hat{g}_{\lambda}\right]\right. \text {. }
$$

Proof. Notice that $\{\langle X, p\rangle,\langle Y, p\rangle\}=-\langle[X, Y], p\rangle$ and $\operatorname{div}[X, Y]$ $=\nabla_{Y} \operatorname{div} X-\nabla_{X} \operatorname{div} Y$, then (2.4) can be verified by using the expression (2.3).

\section{QUANTIZATION ON PRINCIPAL BUNDLES}

In $\S 1$, we constructed the $\lambda$-Weyl transformation on Riemannian manifolds without considering the gauge invariance. Now we generalize it to principal bundles in order to quantize the classical systems in a gauge field [5]. The observables of the quantized systems are differential operators acting on some particle fields which are just wave functions with internal symmetrical structures.

Let $(P, M, \Pi, G)$ be a principal bundle where the base $(M, g)$ is an $n$ dimensional oriented Riemannian manifold. Let $\mathscr{C}$ denote the space of all connections on $P$ which can be considered as a manifold and $G A(P)$, the gauge transformation group on $P$. Assume $V$ to be a complex vector space and the structural group $G$ has a representation in $G L(V)$. It is known that $\Gamma(V)$, 
the space of $C^{\infty}$-sections of the associated bundle $P(V)$ can be considered as $V$-valued functions on $P$ satisfying $\phi(p g)=g^{-1} \phi(p)$ for $p \in P$ and $g \in G$, so we will define the $\lambda$-Weyl transformation on such functions for convenience.

We know that $G A(P)$ acts naturally on $\mathscr{C}$ and $\Gamma(V)$ [1], so it acts on $\mathscr{C} \times \Gamma(V)$ be $\tau^{*}(\theta, \phi)=\left(\tau^{*} \theta, \tau^{*} \phi\right)$ where $\tau \in G A(P), \theta \in \mathscr{C}$ and $\phi \in \Gamma(V)$. In the next paragraph, we will construct the operator $\hat{f}_{\lambda}: \mathscr{C} \times \Gamma(V) \rightarrow \Gamma(V)$ for any polynomial $f$ on $T^{*} M$. We say $\hat{f}_{\lambda}$ is gauge invariant if $\tau^{*} \circ \hat{f}_{\lambda}=\hat{f}_{\lambda} \circ \tau^{*}$, i.e., for any $p \in P$, the following identity holds

$$
\hat{f}_{\lambda}(\theta, \phi)(\tau(p))=\hat{f}_{\lambda}\left(\tau^{*} \theta, \tau^{*} \phi\right)(p) .
$$

This is just the least-coupling principle in physics.

For a fixed connection $\theta \in \mathscr{C}$, let $H_{p}^{\theta} \quad\left(H_{p}^{* \theta}\right)$ denote the $\theta$-horizontal tangent (cotangent) subspace at $p \in P$. By the help of $\theta$, we can lift the exponential map $\exp _{x}: T_{x} M \rightarrow M$ to $\exp _{P}^{\theta}: H_{p}^{\theta} \rightarrow F$, for any $p \in P_{x}$, and lift $f \in$ $C^{\infty}\left(T^{*} M\right)$ to $f^{\theta} \in C^{\infty}\left(T^{*} P\right)$ [5], where $f^{\theta}$ can be considered as the Hamilton function of a classical system moving in the gauge field $P$. In $\S 1$, we have defined the notation $A, \rho_{x}$. They can also be lifted to $P$ naturally and we use just the same notation to express them. From the discussion used in constructing the $\lambda$-Weyl transformation on a Riemannian manifold, we can generalize it naturally to principal bundles as follows:

Definition 3.1. With the notation above, the $\lambda$-Weyl transformation $\hat{f}_{\lambda}$ of a polynomial $f \in C^{\infty}\left(T^{*} M\right)$ is defined as an operator from $\mathscr{C} \times C^{\infty}(P, V)$ to $C^{\infty}(P, V)$ as follows: for any $p \in P$ and $(\theta, \phi) \in \mathscr{C} \times C^{\infty}(P, V)$,

$$
\begin{aligned}
\hat{f}_{\lambda}(\theta, \phi)(p)=h^{-n} \int_{H_{P}^{\theta} \times H_{P}^{* \theta}} f^{\theta}\left(\exp _{P}^{\theta}(\lambda \beta), A(\lambda \beta) \xi\right) & \cdot e^{-i / h\langle\beta, \xi\rangle} \rho_{p}^{1 / 2}\left(\exp _{p}^{\theta} \beta\right) \phi_{\varepsilon}\left(\exp _{P}^{\theta} \beta\right) d \beta d \xi .
\end{aligned}
$$

Remark. Definition 3.1 is a natural generalization of Definition 1.1 in which $G=\{e\}, V=\mathbb{C}, C^{\infty}(P, V)=C^{\infty}(M, \mathbb{C})$ and $\theta$ is the unique flat connection on $P=M \times\{e\}$.

Theorem 3.1. (1) For a fixed $\theta \in \mathscr{C}, \hat{f}_{\lambda}^{\theta} \equiv \hat{f}_{\lambda}(\theta, \cdot)$ is a differential operator with $\Gamma(V) \subset C^{\infty}(P, V)$ as an invariant subspace.

(2) $\left.\hat{f}_{\lambda}\right|_{\mathscr{C} \times \Gamma(V)}$ is gauge invariant, i.e., identity (3.1) holds.

Proof. From the analysis in $\S 1$, it is obvious that $\hat{f}_{\lambda}^{\theta}$ is a differential operator. From facts that, for $\beta \in H_{p}^{\theta}$ and $g \in G,\left(\exp _{p}^{\theta} \beta\right) g=\exp _{p g}^{\theta} g_{*} \beta$ and that $f^{\theta}$ is invariant under the symplectic action $\left(g, g^{-1 *}\right)$ on $T^{*} P$ [5], it is easy to verify that if $\phi(p g)=g^{-1} \phi(p)$, then $\hat{f}_{\lambda}(\theta, \phi)(p g)=g^{-1} \hat{f}_{\lambda}(\theta, \phi)(p)$. Consequently, $\hat{f}_{\lambda}^{\theta}: \Gamma(V) \rightarrow \Gamma(V)$, so (1) is true. To prove (2), let us first prove

Lemma 3.1. For any $\tau \in G A(P)$, the relation $\tau_{*}\left(H_{p}^{\tau^{*} \theta}\right)=H_{\tau(p)}^{\theta}$ holds and, for $\beta \in H_{p}^{\tau^{*} \theta}, \tau\left(\exp _{p}^{\tau^{*} \theta} \beta\right)=\exp _{\tau(p)}^{\theta} \tau_{*} \beta$, where $\tau_{*}$ is the tangent map of $\tau$ at $p$.

Proof. Since $\tau^{*} \theta_{p}=\theta_{\tau(p)}$, i.e., $\tau^{*} \theta_{p}(\beta)=\theta_{\tau(p)}\left(\tau_{*} \beta\right)$ for $\beta \in T_{p} P$, it follows that $\tau_{*}\left(\operatorname{ker} \tau^{*} \theta_{p}\right)=\operatorname{ker} \theta_{\tau(p)}$, i.e., $\tau_{*}\left(H_{p}^{\tau^{*} \theta}\right)=H_{\tau(p)}^{\theta}$. This means $\tau$ transforms a $\tau^{*} \theta$-horizontal curve through $p$ to a $\theta$-horizontal curve through $\tau(p)$, so 
that for $\beta \in H_{p}^{\tau^{*} \theta}$ and $t \in \mathbb{R}$, both $\tau\left(\exp _{p}^{\tau^{*} \theta} t \beta\right)$ and $\exp _{\tau(p)}^{\theta} t \tau_{*} \beta$ are $\theta$ horizontal curves through $\tau(p)$ and they have the same projection to the base $M$. Consequently, they overlap everywhere by the uniqueness of $\theta$-horizontal lift. Setting $t=1$ gives the lemma.

Lemma 3.2. Under the symplectic action $\left(\tau, \tau^{-1 *}\right)$ on $T^{*} P$, the equation

$$
f^{\tau^{*} \theta}(p, \xi)=f^{\theta}\left(\tau(p), \tau_{p}^{-1 *}(\xi)\right)
$$

holds where $\xi \in T^{*} P$.

Proof. This can be verified by Lemma 3.1 and the definition of $f^{\theta}$ [5].

Proof of Theorem 3.1, part (2). From Lemmas 3.1, 3.2 and the fact that the function $\rho$ is constant along each fiber of $P$, identity (3.1) can be verified just by using a linear symplectic isomorphism

$$
\left(\tau_{*}, \tau^{*-1}\right): H_{p}^{\tau^{*} \theta} \times H_{p}^{* \tau^{*} \theta} \rightarrow H_{\tau(p)}^{\theta} \times H_{\tau(p)}^{* \theta}
$$

to expression (3.2).

Formally, Definition 3.1 is same as Definition 1.1, so that it is easy to get

Theorem 3.2. For a fixed $\theta \in \mathscr{C}$ and $f=X^{i j} \xi_{i} \xi_{j}$ we have

$$
\hat{f}_{\lambda}^{\theta}=-h^{2}\left(X^{i j} \nabla_{i}^{\theta} \nabla_{j}^{\theta}+2 \lambda \nabla_{i} X^{i j} \nabla_{j}^{\theta}+\lambda^{2} \nabla_{i} \nabla_{j} X^{i j}-\frac{2 \lambda^{2}+1}{6} X^{i j} R_{i j}\right)
$$

where $\nabla^{\theta}$ is the covariant differential on $\Gamma(V)$ induced by the connection $\theta, \nabla$ is the covariant differential induced by the Levi-Civita connection on tensor fields in the base $M .\left\{R_{i j}\right\}$ is the Ricci curvature tensor. In particular, for $f=\|\xi\|^{2}$,

$$
\hat{f}_{1 / 2}^{\theta}=-h^{2}\left(g^{i j} \nabla_{i}^{\theta} \nabla_{j}^{\theta}-\frac{R}{4}\right) .
$$

Proof. Notice that, for $\phi \in \Gamma(V)$,

$$
\frac{\partial}{\partial \beta_{i}} \phi\left(\exp _{p}^{\theta} \beta\right)=\nabla_{i}^{\theta} \phi \quad \text { and } \quad \frac{\partial}{\partial \beta^{i}} \widetilde{X}\left(\exp _{p}^{\theta} \beta\right)=\nabla_{i} X
$$

where $\tilde{X}$ is the $\theta$-horizontal lift of a tensor field $X$ from $M$ to $P$, then the procedure of proof is like that in Theorem 1.1.

Now we give three examples to illustrate the physical applications of the $\lambda$-Weyl transformation defined for gauge fields. Here the notation follows [1].

Example 3.1 (free electron). Let $M$ be a $2 n$-dimensional spin manifold, $P$ be the spin frame bundle of $M, G=\operatorname{Spin}(2 n), V=\Delta=\Delta^{+} \oplus \Delta^{-}$be the space of spinor representation of $\operatorname{spin}(2 n)$ and $\theta$ be the connection on $P$ induced by the Levi-Civita connection on $M$. In this case, $\Gamma(\Delta)$ is the space of spinor fields (the free Dirac electron fields). Let $\left(e_{1}, \ldots, e_{2 n}\right)$ be a local orthonormal frame, $f=\|\xi\|^{2}$. By expression (3.3) and Lichnerowicz's formula, we have

$$
\hat{f}_{1 / 2}^{\theta}=-h^{2}\left(\nabla_{i}^{\theta} \nabla_{i}^{\theta}-\frac{R}{4}\right)=h^{2} D^{2}
$$

where $D=e_{i} \cdot \nabla_{i}^{\theta}$ is the Dirac operator (with zero charge). 
Example 3.2 (electron in a electromagnetic field). With the same notation $M, G$, $P, V=\Delta$ and $\theta$ as in Example 3.1, now we take $\widetilde{G}=G \times U(1), \widetilde{P}=P \circ P^{\prime}$ as a spliced bundle and $\tilde{\theta}=\theta+\theta^{\prime}$ where $\theta^{\prime}$ is a connection on the $U(1)$-principal bundle $P^{\prime} . \widetilde{V}$ is also taken as $\Delta$, where the representation of $U(1)$ in $G L(\Delta)$ is just scalar multiplication. We know $\theta^{\prime}$ as the electromagnetic gauge potential and $F^{\prime}=\nabla^{\theta^{\prime}} \theta^{\prime}$ as the field strength (curvature). In this case $(U(1)$ is abelian), $F_{i j}^{\prime}=\partial_{i} \theta_{j}^{\prime}-\partial_{j} \theta_{i}^{\prime}$ is a well-defined 2-form on $M$. Through straightforward computation, we have, for $f=\|\xi\|^{2}$,

$$
\hat{f}_{1 / 2}^{\tilde{\theta}}=h^{2}\left(D^{\tilde{\theta}^{2}}+\frac{1}{2} F\right)
$$

where $D^{\tilde{\theta}}=e_{i} \cdot \nabla_{i}^{\tilde{\theta}}=e_{i} \cdot\left(\nabla_{i}^{\theta}+\theta_{i}^{\prime}\right)$ is the charged Dirac operator and $F=F_{i j}^{\prime} e_{i} \cdot e_{j}$ acting on $\Gamma(\Delta)$ by Clifford multiplication.

If a gauge transformation $\tau$ is taken in the form $e^{i s}$ where $s \in C^{\infty}(M, \mathbb{R})$, then (3.1) means

$$
\hat{f}_{\lambda}^{\tilde{\theta}}\left(e^{i s} \phi\right)=e^{i s} \hat{f}_{\lambda}^{\tau^{*} \tilde{\theta}}(\phi)=e^{i s} \hat{f}^{\theta-i d s}(\phi) .
$$

This is a well-known equation in physics.

Example 3.3 (nucleons in a Yang-Mills field). With the same notation as in Example 3.1, now we take $\operatorname{dim} M=4$ (the curved time-space), $\widetilde{G}=\operatorname{spin}(4) \times$ $\mathrm{SU}(2), \widetilde{P}=P \circ P^{\prime}$ and $\tilde{\theta}=\theta+\theta^{\prime}$ where $\theta^{\prime}$ is a connection on the $\mathrm{SU}(2)-$ principal bundle $P^{\prime}$. In this case, $\Delta=\mathbb{C}^{4}$ and we take $\widetilde{V}=\mathbb{C}^{4} \times \mathbb{C}^{4}$. The representation of $\widetilde{G}$ in $G L\left(\mathbb{C}^{4} \times \mathbb{C}^{4}\right)$ can be found in [1]. The Yang-Mills field strength has the form

$$
F_{i j}^{\prime}=\partial_{i} \theta_{j}^{\prime}-\partial_{j} \theta_{i}^{\prime}+\left[\theta_{i}^{\prime}, \theta_{j}^{\prime}\right]
$$

which is su(2)-valued 2-form on $P^{\prime}$. For $f=\|\xi\|^{2}$, it is not difficult to get

$$
\hat{f}_{1 / 2}^{\tilde{\theta}}=h^{2}\left(D^{\tilde{\theta}^{2}}+\frac{1}{2} F\right)
$$

where $D^{\tilde{\theta}}=e_{i} \cdot\left(\nabla_{i}^{\theta}+\theta_{i}^{\prime}\right)$ and $F=F_{i j}^{\prime} e_{i} \cdot e_{j}$. The action of $F$ on $\Gamma\left(\mathbb{C}^{4} \times \mathbb{C}^{4}\right)$ may be found in [1].

\section{APPlications AND REMARKS}

In this section, we show briefly two applications of our quantization rule; the details may be found in [7 and 8]. At the end, some differences of our approach from that of Lichnerowicz [6] and Widom [12] are pointed out.

The first application is about semiclassical approximations of quantized systems on Riemannian manifolds, which is the inverse problem of quantization. In case when $M=\mathbb{R}^{n}$, this can be done to any order of the Planck constant $h$ when $h \rightarrow 0_{+}$since the correspondences between quantum systems and classical systems are already quite clear [9]. But, in general case, if such a correspondence is not clear, some questions will arise from a physical point of view. According to the quantization rule given in this paper, we discussed the semiclassical approximation problem for a quantized system on Riemannian manifolds in [7]. Particularly, in the long time approximation, we set up the semiclassical Green's function to treat the focal problem instead of canonical operators [9] or path-integrations. 
The second one is about quantum integrable systems constrained to the sphere $S^{n-1}$. As usual, we identify the cotangent bundle of $S^{n-1}$ with a symplectic submanifold of $T^{*} \mathbb{R}^{2 n}=\mathbb{R}^{2 n}$ as follows

$$
T^{*} S^{n-1}=\left\{(x, y) \in \mathbb{R}^{2 n} ;\|x\|=1,\langle x, y\rangle=0\right\}
$$

where $\langle\cdot, \cdot\rangle$ denotes the standard inner product in $\mathbb{R}^{n}$. It can also be considered as the orbit space of the Lie group $G=\{(\rho, \alpha) ; \rho>0, \alpha \in \mathbb{R}\}$ acting on $\mathbb{R}^{2 n}$ by

$$
(\rho, \alpha) \cdot(x, y)=(\rho x,(y-\alpha x) / \rho) .
$$

Thus, for every function $f$ on $T^{*} S^{n-1}$, one can get a $G$-invariant function $\bar{f}$ on $R^{2 n}$ (the value at zero may be taken as zero). Let $W(\bar{f})$ denote the Weyl transformation of $\bar{f}$ mentioned in $\S 0$, which is nothing but the operator representation of the invariant star-product on $T^{*} S^{n-1}$ given by Lichnerowicz in [6].

Now, let $g$ denote the Riemannian metric on $S^{n-1}$ induced from $\mathbb{R}^{n}$ by $\langle\cdot, \cdot\rangle$. Thus, for every pointwise polynomial function $f$ on $T^{*} S^{n-1}$, we can get two differential operators, $W(\bar{f})$ and $\hat{f}_{1 / 2}$, acting on $C^{\infty}\left(S^{n-1}\right)$, where $\hat{f}_{1 / 2}$ is the quantization of $f$ on the Riemannian manifold $\left(S^{n-1}, g\right)$ given in this paper. In general, $\hat{f}_{1 / 2} \neq W(\bar{f})$. When $\operatorname{deg} f=2$ as in Theorem 1.1, we get [8]

$$
W(\bar{f})=\hat{f}_{1 / 2}+\frac{3}{2} h^{2} \operatorname{tr} X, \quad \operatorname{tr} X=X^{i j} g_{i j} .
$$

In particular, for $H$ being the Hamiltonian of the geodesic flow on $\left(S^{n-1}, g\right)$, (4.1) becomes

$$
W(\bar{H})=-h^{2}\left(\Delta+c_{n} R\right) .
$$

Compared with what was mentioned in $\S 0$, it seems that $W(\cdot)$ is unsuitable to be a quantization rule on $T^{*} S^{n-1}$, for the constant $c_{n}$ depends on the dimensions of the phase spaces. In [8], we showed that the quantized geodesic flow on $S^{n-1}$, and the C. Neumann system (i.e., harmonic oscillator constrained to the sphere) are quantum integrable by use of our quantization rule and formula (4.1).

Finally, we noticed that Widom set up a correspondence between pseudodifferential operators and their symbols on Riemannian manifolds in [12] without paying attention to the physical aspects. To see the relation between his approach and ours, let us first write down an explicit expression for the phase function give in [12] near each point in $M$. Let $U_{2 \varepsilon}$ be a small ball in $M$ with center $x_{0}$ such that, for any $x \in U_{\varepsilon}, y \in U_{2 \varepsilon}$, the map $\exp _{x}^{-1}(y) \in T_{x} M$ is well defined. Thus the function given by

$$
L(p, y)=\left\langle p, \exp _{x}^{-1}(y)\right\rangle, \quad p \in T_{x}^{*} M, y \in U_{2 \varepsilon},
$$

is well defined on $T^{*} U_{\varepsilon} \times U_{2 \varepsilon}$, and satisfies conditions of the phase function near $x_{0}$, which can be checked by taking normal coordinates at each point in $U_{\varepsilon}$. For a differential operator $A$, its symbol given by Widom is

$$
\sigma_{A}(x, p)=\left.A e^{i L(p, y)}\right|_{y=x} .
$$

By definition of our normal ordered $(\lambda=0)$ quantization, it is easy to see that, for any $\varphi \in C^{\infty}(M)$, one has (take $h=1$ ),

$$
\left(\left(\widehat{\sigma_{A}}\right)_{0} \varphi\right)(x)=A\left(\rho_{x}^{1 / 2} \varphi\right)(x)
$$


where $\rho_{x}$ was introduced in Step 3 of our definition in order to get a unitary transformation, so Widom actually treated the case corresponding to $\lambda=0$.

\section{REFERENCES}

1. D. Bleecker, Gauge theory and variational principles, Addison-Wesley, 1981.

2. J. Czyz, On geometric quantization and its connection with Maslov theory, Rep. Math. Phys. 15 (1979), 57-97.

3. C. De Witt-Morette and D. Elworthy, A stochastic scheme for constructing solutions of Schrödinger equations, Ann. Inst. H. Poincaré A-32 (1980), 327-341.

4. D. Elworthy, Path integrations on manifolds, Mathematical Aspects of Superspace, Reidel, 1984.

5. V. Guillemin and S. Sternberg, Symplectic techniques in physics, Cambridge Univ. Press, 1984.

6. A. Lichnerowicz, Twisted products for cotangent bundles of classical groups, Lett. Math. Phys. 2 (1977), 133-143.

7. Z. J. Liu, Semi-classical approximations on Riemannian manifolds, Acta Appl. Math. Sinica (to appear).

8. __ Quantum integrable systems constrained on the sphere, Lett. Math. Phys. 20 (1990), 151-157.

9. V. P. Maslov and M. P. Fedoriuk, Semi-classical approximation in quantum mechanics, Reidel, 1981.

10. J. Underhill, Quantization on a manifold with connection, J. Math. Phys. 19 (1978).

11. A. Weinstein, Quasi-classical mechanics on spheres, Istituto Nazionale di Mat. Sympos. Math. 14 (1974).

12. H. Widom, A Szego theorem and symbol calculus for pseudo differential operators, Ann. of Math. Stud., no. 91, Princeton Univ. Press, 1979, pp. 261-283.

13. E. Wolf and G. Agarwal, Calculus for functions of non-commuting operators, Phys. Rev. D 12 (1970), 2161-2186.

14. N. Woodhouse, Geometric quantization, Oxford Univ. Press, 1980. CHINA

Department of Mathematics, Peking University, Beijing 100871, Peoples Republic of 